\title{
Kerstin Peglow
}

Université Paris Ouest Nanterre La Defense e-mail: kpeglow@u-paris10.fr

\section{Entstehung und Geschichte der Gesellschaft mit beschränkter Haftung im deutschen Recht}

\author{
SUMMARY \\ Origin and History of the German Gesellschaft mit beschränkter Haftung \\ (Private Limited Company)
}

The Gesellschaft mit beschränkter Haftung - GmbH - is a widespread company form, known all over the world, which was adopted in 1892 by the German legislator. Its historical origins were essentially favored by important economic growth during the second half of the 19thcentury. Smaller and medium-sized enterprises should be able to choose the legal form of the Gesellschaft mit beschränkter Haftung in order to participate in a more convenient and easily accessible way in the country's economic growth. This purpose could not be achieved by the highly regulated Aktiengesellschaft (public limited company) which was dedicated to large companies and was therefore too expensive and too complicated for smaller entities. Partnerships like, for instance, the Offene Handelsgesellschaft (general partnership) had the disadvantage that all partners were liable without restrictions to its creditors. The Gesellschaft mit beschränkter Haftung was thought to cover the gap between corporations and partnerships.

Despite some weaknesses, the Gesellschaft mit beschränkter Haftung subsequently became a real model of success. Over the years, the Act on the $\mathrm{GmbH}$ was subject to constant reforms through practice and legislation, because it was necessary to take into account economic requirements and to counter maladministration in application of the $\mathrm{GmbH}$ Law. More recently, European legal standards had a further significant influence on the Gesellschaft mit beschränkter Haftung. Since its creation, the $\mathrm{GmbH}$ has played a key role in German legal and economic life. It does exist in many diverse forms and has been for a long time the most popular form of company in Germany.

Key words: GmbH, commercial law, limited liability company, joint stock company 


\section{Die Einführung der GmbH im Jahre 1892}

Im ausgehenden 19. Jahrhundert entstand in Deutschland eine neue Rechtsform, die Gesellschaft mit beschränkter Haftung. Sie war eine ureigene Schöpfung des Gesetzgebers losgelöst von jedem geschichtlichen Vorbild ${ }^{1}$. Die Schaffung der $\mathrm{GmbH}$ erklärt sich aus der damals bestehenden wirtschaftlichen Lage und dem zu jener Zeit geltenden Gesellschaftsrecht. Die Notwendigkeit über den Sinn einer neuen Gesellschaftsform zur Durchsetzung bestimmter wirtschaftspolitischer Ziele löste intensive Diskussionen unter Vertretern von Recht, Politik und Wirtschaft aus. Nach umfassenden Stellungnahmen der verschiedenen deutschen Handelskammern wurde am 26. April 1892 das Gesetz betreffend die Gesellschaft mit beschränkter Haftung verabschiedet.

\section{Die Gründe für die Schaffung der neuen Rechtsform zum Ende des 19. Jahrhunderts}

\section{a) Wirtschaftliche Situation}

Die Entstehung der GmbH als neuer Gesellschaftsform ist entscheidend durch das bedeutsame Wirtschaftswachstum in Deutschland zum ausgehenden 19. Jahrhundert begünstigt worden. Im Zuge der Gründung des deutschen Reiches im Jahre 1871 war ein großer Markt geschaffen worden, die Infrastruktur wurde ausgebaut und bereits 1869/70 war die Gewerbefreiheit eingeführt worden, sodass die industrielle Revolution sich voll entfalten konnte. Der Auftrieb der deutschen Wirtschaft beruhte außerdem, wenn auch nur bis zum Herbst 1873, auf den hohen französischen Kriegsentschädigungen, die verstärkt $\mathrm{zu}$ Unternehmensgründungen führten. Ab 1873 bis 1895 folgte eine Phase verlangsamten Wachstums, die jedoch insgesamt die wirtschaftliche Entwicklung nicht aufhielt ${ }^{2}$.

1 Münchener Kommentar zum Gesetz betreffend die Gesellschaft mit beschränkter Haftung - GmbHG, Band 1, $\$ 1-34$, herausgegeben von Holger Fleischer und Wulf Goette, Verlag C. H. Beck, München 2010, Einl. (Holger Fleischer), Rn 50, Zitierweise: MünchKommGmbHG/Fleischer; K. Schmidt, Gesellschaftsrecht, 4. Auflage, Carl-Heymanns Verlag, Köln, Bonn, Berlin, München 2002, S. 986, Zitierweise: K. Schmidt, Gesellschaftsrecht; A. Baumbach, A. Hueck, GmbHG, Gesetz betreffend die Gesellschaften mit beschränkter Haftung, 19. Auflage, Verlag C.H. Beck, München 2010, Einl. (Goetz Hueck/Lorenz Fastrich) Rn 18, Zitierweise: Baumbach/Hueck, GmbHG, Hueck/Fastrich; J. Meyer, Haftungsbeschränkung im Recht der Handelsgesellschaften, Springer-Verlag Berlin, Heidelberg, New York, 2000, S. 289 f., Zitierweise: J. Meyer.

2 Münchener Handbuch des Gesellschaftsrechts, Band 3, Gesellschaft mit beschränkter Haftung, herausgegeben von Hans-Joachim Priester und Dieter Mayer, Verlag C.H. Beck München 2003, $\$ 1$ (Herbert Grziwotz) Rn 2, Zitierweise: Münch. Handbuch, GmbH/Grziwotz; J. Meyer, S. 290. 
Parallel $\mathrm{zu}$ den wirtschaftlichen Fortschritten war auch ein hohes Bevölkerungswachstum zu verzeichnen. Zwischen 1850 und 1880 stieg die Bevölkerung von 34 Millionen um mehr als ein Drittel, bis 1890 betrug sie fast 50 Millionen. Diese Zunahme war nicht nur auf Gebietszuwächse zurückzuführen, sondern beruhte $\mathrm{zu}$ einem erheblichen Teil auf dem immer breitere Bevölkerungsschichten erfassenden Wohlstand und der sehr bedeutsamen Steigerung des Pro-Kopf Einkommens. Der sich darin widerspiegelnde Wandel zur Industriegesellschaft zeigte sich auch in der Verlagerung der Beschäftigungsverhältnisse. War 1870 noch rund die Hälfte der erwerbstätigen Bevölkerung in der Landwirtschaft tätig, war es 1910 kaum noch ein Drittel. Bereits 1890 wurden damit einhergehend im industriellen Sektor mehr Einnahmen erzielt als in der landwirtschaftlichen Produktion ${ }^{3}$.

Aufgrund dieser positiven gesamtwirtschaftlichen Situation kam es in den Jahren vor der Schaffung der $\mathrm{GmbH}$ zu einem Kapitalüberschuss, der, wie seinerzeit bemängelt wurde, ins Ausland abgezogen wurde. Diesem Zustand wollte man ein Ende bereiten, indem investitionsfreudigen Unternehmern eine rechtliche Lösung angeboten werden sollte, ihr Kapital einfach und gewinnbringend im Inland zu binden ${ }^{4}$.

\section{b) Stand des Gesellschaftsrechts}

Als Anlage- und Unternehmensform diente seinerzeit insbesondere die Aktiengesellschaft. Deren Regeln über Gründung und Verwaltung waren jedoch durch die Aktienrechtsnovelle von 1884 erheblich verschärft worden, da die 1870 liberalisierten Vorschriften des Aktiengesetzes während der Gründerzeit zu schweren Missbräuchen geführt hatten ${ }^{5}$. Die Aktiengesellschaft eignete sich aufgrund ihrer umfangreichen Schutzvorschriften daher vorwiegend für große Unternehmen, die sich wegen ihres Kapitalbedarfs an ein breites Publikum wendeten. Kleinere und mittlere Unternehmen mit einer beschränkten Zahl von Mitgliedern, die sich untereinander kannten und an der Gesellschaft aktiv teilnehmen wollten, konnten durch diese Rechtsform nicht hinreichend zufriedengestellt werden. Für sie wurde die Aktiengesellschaft zu teuer und zu schwerfällig ${ }^{6}$.

Als Alternative zur Aktiengesellschaft boten sich für mittelständische Unternehmen die traditionellen Rechtsformen der Offenen Handelsgesellschaft

\footnotetext{
Münch. Handbuch, GmbH/Grziwotz, §1, Rn 2; J. Meyer, S. 290 f.

Ibidem, S. 291.

Gesetz, betreffend die Kommanditgesellschaft auf Aktien und die Aktiengesellschaften vom 18.7.1884, RGBl. (Reichsgesetzblatt) S. 123. Verschärft worden war insbesondere das Gründungsrecht durch neue Regeln zur Gründungsprüfung, Gründerhaftung und der Publizität der Gründung

6 Baumbach/Hueck, GmbHG, Hueck/Fastrich, Einl., Rn 19; Münch. Handbuch, GmbH/Grziwotz, $\$ 1$, Rn 7.
} 
oder der Kommanditgesellschaft an. Diese hatten jedoch den Nachteil der unbeschränkten Haftung der Gesellschafter, sodass sie nicht wirklich den bestehenden Erfordernissen für eine moderne Unternehmensform gerecht werden konnten ${ }^{7}$.

Auch eine Lösung wie sie für die damals existierenden Kolonialgesellschaften im Jahre 1888 gefunden wurde, lie $\beta$ sich auf die inländischen Verhältnisse nicht übertragen. Die Kolonialgesellschaften unterlagen keiner besonderen Rechtsform ${ }^{8}$. Sie lehnten sich vielfach in ihrer Struktur an das Aktienrecht an, enthielten teilweise aber auch Regelungen des Personengesellschaftsrechts. Gegen eine vollständige Übernahme des Aktienrechts sprachen verschiedene Gründe, die sich unter anderem aus der geographischen Entfernung ergaben. Wichtig war für die Kolonialgesellschaften vor allem die Haftungsbeschränkung wegen der häufig unsicheren Lage in den Kolonien. Diese wurden ihnen durch ein Gesetz von 1888 durch die Erteilung der Rechtsfähigkeit zugestanden, ohne dass jedoch gleichzeitig die Organisationsform der Kolonialunternehmungen geregelt wurde. Solch eine pragmatische Lösung war für die relative kleine Anzahl von betroffenen Gesellschaften angemessen, konnte jedoch nicht dazu dienen, das heimische Bedürfnis nach einer haftungsbeschränkenden Gesellschaftsform zu befriedigen. Immerhin gaben diese Regelungen zusätzliche Anstöße über dahingehende Reformen nachzudenken ${ }^{9}$.

Die Schwierigkeiten des geltenden Gesellschaftsrechts potentiellen Gesellschaftsgründern eine adäquate Lösung anzubieten, lie $\beta$ den Ruf nach einer neuen Gesellschaft mit Haftungsbeschränkung aufkommen ${ }^{10}$. Sie sollte die Lücke zwischen der Aktiengesellschaft als Kapitalgesellschaft und den Personenhandelsgesellschaften schließen und einen Mittelweg zwischen diesen bereits bekannten Gesellschaftsformen darstellen ${ }^{11}$.

Baumbach/Hueck, GmbHG, Hueck/Fastrich, Einl., Rn 20.

J. Meyer, S. 294 f.

9 J. Meyer, S. 294 f.; MünchKommGmbHG/Fleischer, Einl., Rn 53; Werner Schubert, Das GmbH-Gesetz von 1892 „eine Zierde unserer Reichsgesetzsammlung”, [in:] Festschrift 100 Jahre GmbHGesetz, herausgegeben von Marcus Lutter, Peter Ulmer, Wolfgang Zöllner, Verlag Dr. Otto Schmidt, Köln 1992, S. 4 ff., Zitierweise: W. Schubert, GmbHG 1892.

10 K. Schmidt, Gesellschaftsrecht, S. 986; Münch. Handbuch, GmbH/Grziwotz, § 1, Rn 11.

11 Baumbach/Hueck, GmbHG, Hueck/Fastrich, Einl., Rn 20; W. Schubert, S. 8. 


\section{Das Gesetz betreffend die Gesellschaften mit beschränkter Haftung von 1892}

\section{a) Reformvorschläge und Diskussion}

Die ersten Forderungen nach einer neuen Gesellschaftsform wurden bereits im Jahr 1884 laut anlässlich der Beratungen zur Aktienrechtsnovelle ${ }^{12}$. Während der sich über mehrere Jahre erstreckenden rechtspolitischen Diskussion wurden verschiedene Reformvorschläge erarbeitet, wobei sich im Wesentlichen zwei Lösungsansätze unterscheiden ließen.

Auf der einen Seite standen die Befürworter einer „Offenen Handelsgesellschaft mit beschränkter Haftung“. Ihr bekanntester Vertreter war der Industrielle und nationalliberale Reichstagsabgeordnete Wilhelm OechelhäUser, der häufig als der "geistige Vater" der GmbH genannt wird ${ }^{13}$. Nach den von dieser Ansicht präsentierten Vorschlägen sollte eine neue Gesellschaftsform auf der Basis der Offenen Handelsgesellschaft eingeführt werden, bei der aber die solidarische Haftung der Gesellschafter auf bestimmte Kapitaleinlagen beschränkt war. In den Einzelheiten wichen die Vorschläge hingegen voneinander ab. Uneinigkeit bestand bei den Verfechtern dieser „individualistischen“ Lösung unter anderem darüber, ob ein Mindestkapital für die Gesellschaft gesetzlich festzulegen sei oder nicht ${ }^{14}$.

Die Gegenansicht bevorzugte die "kollektivistische“ Lösung, wonach die neue Rechtsform als „vereinfachte“ Aktiengesellschaft $\mathrm{zu}$ konzipieren sei. Zum einen sollten die strengen Gründungsvorschriften der Aktiengesellschaft nicht zur Anwendung kommen, zum anderen sollten die Anteile schwerer übertragbar sein. Als Modell wurde dabei an die Regelungen der bergrechtlichen Gewerkschaft gedacht ${ }^{15}$.

Am 3. April 1988 richtete das preußische Ministerium für Handel und Gewerbe auf Veranlassung des Reichsamts des Innern an sämtliche Handelskammern und den Deutschen Handelstag eine Anfrage über den Bedarf in Handel und Industrie an einer neuen Rechtsform. In den 81 eingegangenen Stellungnahmen äußerten sich die Handelskammern überwiegend zustimmend, obwohl dies teilweise im Widerspruch zum

12 W. Schubert, GmbHG 1892, S. 4 ff.

13 J. Meyer, S; 296 mit weiteren Nachweisen; Abdruck des Entwurfs von Oechelhäuser bei Wolfgang Schilling, Rechtspolitische Gedanken zur GmbH\&Co., [in:] Festgabe für Otto Kunze, Recht und Rechtsleben in der sozialen Demokretie, Dunckler \& Humbolt, Berlin 1969, S.189/205 ff., siehe auch MünchKommGmbHG/Fleischer, Einl., Rn 55 ff.

14 J. Meyer, S. 296.

15 Bekanntester Befürworter dieser Lösung war das langjährige Mitglied des Reichstages Friedrich Adolf Hammacher, vgl. zu seinem und anderen Vorschlägen die Angaben bei W. Schubert, GmbHG 1892, S. 14 ff. und bei J. Meyer, S. 297 ff. 
ermittelten Bedarf in den jeweiligen Kammergebieten stand ${ }^{16}$. Von der Sache her wurde mehrheitlich der „individualistischen“ Lösung der Vorzug gegeben. Der Deutsche Handelstag schloss sich diesem Vorschlag im Grundsätzlichen an und legte dazu einen von ihm gebilligten Entwurf bei, der sich eng an den Vorschlag von Oechelhäuser anlehnte ${ }^{17}$.

Seitens der Handelskammern wurde ein konkretes Bedürfnis zur Schaffung einer neuen Gesellschaftsform besonders in vier Bereichen gesehen. Dabei handelte es sich um landwirtschaftliche Nebenerwerbsgesellschaften wie die Zuckerrübenfabriken, Versuchsgesellschaften zur Patentverwertung, Gläubigergesellschaften zur Sanierung einer in Schwierigkeiten geratenen Gesellschaft sowie um die Übernahme eines Geschäfts durch die Erben. Im Interesse der Arbeitnehmer und wegen des Engagements der Familienmitglieder sei eine Weiterführung des Betriebs wünschenswert, scheitere aber häufig daran, dass die Erben das Risiko der unbeschränkten Haftung nicht tragen wollten ${ }^{18}$.

Ein weiteres Argument sahen die Befürworter der Einfügung einer neuen Rechtsform in den zu beobachtenden Missbräuchen bei der Aktiengesellschaft. Kritisiert wurde, dass viele Gesellschaften, für die die Aktiengesellschaft ihrer Natur nach absolut nicht passe, dennoch diese Form angenommen hätten, weil eben keine andere Gesellschaftsform zur Verfügung stände. Als Beispiel wurden u. a. genannt religiöse Vereine, die lediglich die Krankenpflege oder die Verbreitung von Flugblättern zum Inhalt hatten, studentische Vereinigungen, die ihr Gesellschaftshaus finanzieren wollten, oder gewöhnliche Studentenkneipen. Zusätzlich beklagte man auch die Verwendung der bergrechtlichen Gewerkschaft, die, obwohl nur für bergrechtliche Zwecke gedacht, oft anstatt der komplizierten Aktiengesellschaft gewählt wurde ${ }^{19}$.

Mit Blick über die Grenzen zog man außerdem zum Vergleich die Situation der englischen „limited companies” heran. Die Existenz dieser einfachen und sicheren Rechtsform, die jedermann Haftungsbeschränkung gewähre, würde für England im Wettbewerb einen wirtschaftlichen Vorteil bedeuten. Die „limited” könne aufgrund ihrer „unangemessenen“ Vermehrung und ihrer Verwendung für Geschäfte von unbedeutendem Umfang als Argument für die Effektivität solcher haftungsbeschränkenden Gesellschaftsformen dienen ${ }^{20}$.

1652 Gutachten stimmten der Einführung einer neuen Gesellschaftsform zu, wobei 19 sowohl für eine kollektivistische als auch für eine individualistische Gesellschaft, 20 für eine individualistische und der Rest für eine kollektivistische Gesellschaft stimmten. 29 Gutachten lehnten die Schaffung einer weiteren Gesellschaftsform ab (vgl. W. Schubert, GmbHG 1892, S. 10).

17 MünchKommGmbHG/Fleischer, Einl., Rn 62 f.; Münch. Handbuch, GmbH/Grziwotz, § 1, Rn 12.

18 J. Meyer, S. $301 \mathrm{f}$.

19 W. Schubert, GmbHG 1892, S.14; J. Meyer, S. 293.

20 J. Meyer, S. 293; W. Schubert, GmbHG 1892, S. 5; MünchKommGmbHG/Fleischer, Einl., Rn 217, mit Hinweis auf den Entwurf eines Gesetzes betreffend die Gesellschaften mit beschränkter Haftung. 
Die Diskussion im Reichstag und den verschiedenen handelsrechtlichen Organisationen machte insgesamt deutlich, dass eine Erweiterung der bestehenden Rechtsformen gewünscht wurde. Dabei standen die wirtschaftlichen Interessen von Handel, Industrie und Staat im Vordergrund. Wichtig erschien vor allem die Haftungsbeschränkung der gesellschaftlichen Beteiligung. Auf der Grundlage der Reformvorschläge und der Umfrageergebnisse erfolgte daher im Reichsjustizamt 1890/1891 die Ausarbeitung eines Gesetzesentwurfes ${ }^{21}$.

\section{b) Gesetzesentwurf und Verabschiedung des Gesetzes}

Der vom zuständigen Referenten im Dezember 1890 vorgelegte Gesetzesentwurf wich erheblich von den bisherigen Vorschlägen ab, stimmte aber weitgehend mit dem späteren Gesetz überein. Möglich erscheint, dass sich der im Reichsjustizamt mit der Angelegenheit betraute Sachbearbeiter von dem sehr eigenständigen Vorschlag der Handelskammer Saarbrücken hatte leiten lassen ${ }^{22}$. Im Einzelnen ging der Entwurf davon aus, dass die Abschaffung der unbeschränkten Haftung für die neue Gesellschaftsform als unvereinbar mit dem Charakter einer Personenhandelsgesellschaft anzusehen sei. Die Regelung der zu schaffenden Rechtsform sollte in einem Sondergesetz niedergelegt werden ohne Verweisung auf das Recht des damals geltenden Handelsgesetzbuchs und das Recht der Offenen Handelsgesellschaft. Insgesamt sah der Gesetzesentwurf vor, die Prinzipien des Rechts der Aktiengesellschaft als Vorbild zu nehmen. Dabei sollte die neue Gesellschaft entschieden „individualistischer konzipiert, gleichzeitig aber auch für die Aufnahme einer größeren Zahl von Mitgliedern geeignet und deshalb wie die Aktiengesellschaft korporativ organisiert sein. „Die Gesellschaft sollte für jeden gesetzlich zulässigen Zweck gegründet werden können, der Gründungsvertrag unterlag der Schriftform. Die Übertragbarkeit der Anteile wurde an die Beachtung von Formvorschriften geknüpft, um $\mathrm{zu}$ verhindern, dass sie zum Gegenstand des freien Handelsverkehrs gemacht würden. Bei der Ausgestaltung des Innenverhältnisses wurde den Gesellschaftern ein großer Freiraum gelassen ${ }^{23}$. Während der folgenden parlamentarischen Beratungen wurden nur unwesentliche Änderungen des Entwurfs vorgenommen ${ }^{24}$.

21 Münch. Handbuch, GmbH/Grziwotz, §1, Rn 12.

22 Ausführlich zum Inhalt des Entwurfs der Handelskammer Saarbrücken: W. Schubert, S. $10 \mathrm{ff.}$

23 Vgl. die Zitate aus dem Entwurf in MünchKommGmbHG/Fleischer, Einl., Rn 64 und seine ausführliche Darstellung bei W. Schubert, GmbHG 1892, S. 22 ff. sowie bei J. Meyer, S. 305 f. und Münch. Handbuch, GmbH/Grziwotz, $\$ 1$, Rn 13.

24 So wurde z.B. wegen der im Gesellschaftsvertrag vorgesehenen Gestaltungsfreiheit, die notarielle oder gerichtliche Beurkundung festgelegt (Münch. Handbuch, GmbH/Grziwotz, $\ 1$, Rn 15; W. Schubert, GmbHG 1892, S. 29). 
Nachdem der Entwurf im Juli 1891 vom Kaiser gebilligt worden war, wurde er wenig später unter dem Titel „Entwurf eines Gesetzes, betreffend die Gesellschaften mit beschränkter Haftung" veröffentlicht. Nach Zustimmung des Bundesrates brachte die Reichsregierung den Entwurf in den Reichstag ein, der darüber ab dem 19. Februar 1892 unter Einbeziehung einer vierzehnköpfigen Kommission in drei Lesungen beriet. Ohne größere parlamentarische Debatte wurde die nur unwesentlich geänderte Gesetzesfassung am 21. März 1982 in dritter Lesung angenommen. Die Zustimmung des Bundesrates erfolgte am 7. April 1892. Nach der Ausfertigung durch den Kaiser am 20. April 1892 wurde das Gesetz am 26. April im Reichsgesetzblatt verkündet und trat am 10. Mai 1892 in $\mathrm{Kraft}^{25}$.

Das Gesetz folgte im Grundsatz dem „kollektivistischen” Modell ${ }^{26}$. Das ergibt sich aus der Gesetzesbegründung in der es hei $\beta$ t, dass die Offene Handelsgesellschaft aufgrund ihrer individualistischen Grundlage nur für eine sehr geringe Zahl von Gesellschaftern die geeignete Beteiligungsform sei. Auch würde die Anlehnung an die Offene Handelsgesellschaft den Eindruck erwecken, die Personen würden bei der neuen Gesellschaftsform im Vordergrund stehen, obwohl die Gesellschafterpflichten auf die Leistung der Einlage beschränkt seien. Die Verfasser des Gesetzes hoben hervor, dass die neue Gesellschaftsform einen rechtlich selbständigen Organismus ohne Rücksicht auf die Zahl der Mitglieder darstellte. Daher sei von derselben allgemeinen Grundlage auszugehen wie bei der Aktiengesellschaft. Gleichzeitig führe aber die inhaltliche Gestaltungsfreiheit der Gesellschafter dazu, dass die Gesellschaft eine Mittelstellung zwischen streng individualistischen Gesellschaften und den entschieden kapitalistisch geprägten Aktiengesellschaften einnehme ${ }^{27}$. Diese Deutung des Gesetzes wurde im Weiteren auch vom zeitgenössischen und modernen Schrifttum vertreten.

\section{Die rechtliche Würdigung des GmbH-Gesetzes und seine Umsetzung in die Praxis}

\section{a) Zustimmung und Kritik}

In der Praxis und in der Rechtspolitik wurde das neue GmbH-Gesetz durchweg positiv aufgenommen. Eine Gesellschaftsform des „kleinen Mannes“ sei geschaffen worden und die bestehende Lücke zwischen Kapital- und Personengesellschaften würde nun nicht mehr bestehen ${ }^{28}$.

25 Münch. Handbuch, GmbH/Grziwotz, \$1, Rn 16; MünchKommGmbHG/Fleischer, Einl., Rn 65, 66. Siehe zu den Beratungen im Bundesrat und im Reichstag: W. Schubert, GmbHG 1892, S. 27 ff.

26 MünchKommGmbHG/Fleischer, Einl., Rn 73.

27 Zur Gesetzesbegründung siehe MünchKommGmbHG/Fleischer, Einl., Rn 71, 72.

28 So der Abgeordnete Oechelhäuser laut der Stenographischen Berichte des Reichstages, 1892, zitiert in MünchKommGmbHG/Fleischer, Einl., Rn 76. 
Eine Besonderheit bei der Ausarbeitung des Gesetzes beruhte darauf, dass die Vertreter der Rechtswissenschaft während des schnell durchgezogenen Gesetzgebungsverfahrens praktisch keine Gelegenheit erhalten hatten, sich zu äußern. Die Einführung der $\mathrm{GmbH}$ war auschließlich als das Werk gesellschaftsrechtlich erfahrener Praktiker anzusehen. Die dementsprechend erst zum Ende des Verfahrens im Schrifttum aufkeimende Kritik rügte, dass die beschränkte Haftung voraussichtlich zu einem "Schwindel ohne gleichen” zulasten der Gläubiger führen würde ${ }^{29}$. Die Gläubigersicherung sei durch das Stammkapital völlig unzureichend garantiert und würde die erst durch die Aktienrechtsnovelle veranlassten Sicherungsmechanismen ins Leere laufen lassen. Andere bemängelten, dass durch die beschränkte Haftung prinzipiell solidere Rechtsformen wie die Offene Handelsgesellschaft und die Kommanditgesellschaft verdrängt werden würden. Die Haftungsbeschränkung sei generell nicht gerechtfertigt in einer Gesellschaft, in der die Gesellschafter die Geschäfte selbst führten und somit die notwendige Aufsicht sicherstellen könnten ${ }^{30}$.

Die Kritik erwies sich später zum Teil als fundiert, in anderen Teilen wurde sie durch den großen Erfolg der GmbH in der Praxis widerlegt.

\section{b) Erste Erfahrungen nach Inkrafttreten des Gesetzes}

In den ersten Jahren nach Inkrafttreten des neuen Gesetzes verlief die Gründung von Gesellschaften mit beschränkter Haftung recht zögerlich, was damit begründet wird, dass zunächst Unsicherheiten und mangelnde Erfahrungswerte bei der Anwendung des neuen Rechts bestanden ${ }^{31}$. Die Statistiken jener Zeit zu der Anzahl der Neugründungen und den einzelnen Daten der Gesellschaft waren zunächst sehr detailliert. Im Jahr 1892 wurden 63 Gesellschaften gegründet, 1897 waren es bereits 640 und im April 1902 konnten insgesamt 4745 verzeichnet werden. Ein knappes Viertel der bis dahin gegründeten 6200 Gesellschaften war durch Konkurs, Liquidation oder Gesellschaftsformwechsel wieder gelöscht worden. Beachtlich ist auch, dass es bereits 1893 mehr Gründungen von Gesellschaften mit beschränkter Haftung als von Aktiengesellschaften gab. Ende 1914 standen 27000 Gesellschaften mit beschränkter Haftung 5500 Aktiengesellschaften gegenüber. Nach den Aufzeichnungen wurde die $\mathrm{GmbH}$ in steigendem Maße von kleineren Unternehmen gewählt. Familienunternehmen, Einmanngesellschaften, Kolonial-

29 Vgl. die geäußerte Kritik von Otto Bähr in seiner Schrift „Die Grenzboten” von 1892, zitiert bei J. Meyer, S. 304.

30 In diesem Sinne die Kritik von Levin Goldschmidt, „Alte und neue Formen der Handelsgesellschaften", in Vermischte Schriften, Band II, zitiert bei J. Meyer, S. 305, W. Schubert, GmbHG 1892, S. 2 und MünchKomm GmbHG/Fleischer, Einl., Rn 79.

31 Münch. Handbuch, GmbH/Grziwotz, \$1, Rn 6; einschränkend W. Schubert, GmbHG 1892, S. 43. 
gesellschaften oder Patentverwertungsgesellschaften verwendeten häufig die Rechtsform der $\mathrm{GmbH}$, aber auch alle anderen denkbaren Industrie- und Handelsbetriebe machten von ihr Gebrauch ${ }^{32}$.

Die anfänglich geäußerten Zweifel an der finanziellen Belastbarkeit der $\mathrm{GmbH}$ bestätigten sich zunächst nicht, erst nach 1905 stieg die Zahl der Konkurse an. Kritisiert wurde in der nur spärlichen Literatur zur GmbH vor allem die Umgehung der Vorschriften zum Stammkapital durch Einbringung minderwertiger Sacheinlagen ${ }^{33}$. Ansonsten wurde von ihr sowohl in rechtlicher als auch in tatsächlicher Hinsicht ein durchaus positives Bild gezeichnet ${ }^{34}$.

\section{Die weitere Entwicklung des GmbH-Rechts}

Wurde unmittelbar nach Schaffung der neuen Rechtsform auch keine beeindruckende Gründerwelle ausgelöst, so hatte die $\mathrm{GmbH}$ doch in der Folgezeit bis zur Gegenwart einen enormen Erfolg zu verzeichnen. Daran ändert auch die Tatsache nichts, dass das $\mathrm{GmbH}$-Recht seit seiner Entstehung von ständigen Rufen nach Reformen begleitet wurde, die zum Teil auch zu Änderungen führten ${ }^{35}$. Entscheidend mitgeprägt wurde das Bild der heutigen $\mathrm{GmbH}$ durch die praktische Rechtsfortbildung. Anpassungen des GmbH-Gesetzes sind zudem im Zuge der europäischen Rechtsangleichung notwendig geworden.

\section{Die GmbH im Wandel des 20. Jahrhunderts}

\section{a) Reformansätze und Rechtsfortbildung}

Das GmbH-Recht ist von wiederholten Reformdiskussionen gekennzeichnet, die ein Zeugnis für die Schwachpunkte der $\mathrm{GmbH}$ ablegen. Vielfach verblieb es aber bei Reformansätzen und gesetzliche Abänderungen fanden nicht statt. Einige Reformdebatten sind besonders hervorzuheben.

Bereits am Anfang des 20. Jahrhunderts forderten Stimmen in der Literatur, die $\mathrm{GmbH}$ einer gemäßigten Revision zu unterziehen. Der Gläubigerschutz und die Kreditwürdigkeit sollten durch eine strengere Haftung der Gründer

32 Ausführlich zur Verbreitung und den Verwendungszwecken der GmbH, W. Schubert, GmbHG 1892, S. $30 \mathrm{ff}$.

33 Vgl. die Kritik von Neukamp, Die deutschen Gesellschaften mit beschränkter Haftung, eine neue Gesellschaftsform, Zeitschrift für Volkswirtschaft, Socialpolitik und Verwaltung, Band 8, 1898, zitiert bei J. Schubert, S. 30 f. Das Stammkapital musste damals mindestens 20.000 Mark betragen (W. Schubert, GmbHG 1892, S. 33).

34 W. Schubert, GmbHG 1892, S. 41 mit Nachweisen zur zeitgenössischen Literatur.

$35 \mathrm{Zu}$ den seit jeher dauernden Diskussionen um die GmbH: Kurt Ballerstedt, 75 Jahre GmbH-Gesetz, GmbHR, 1978, S. 66-69. 
und der Gesellschafter verstärkt werden ${ }^{36}$. Andere sprachen sich für insgesamt schärfere Gründungsregeln aus ${ }^{37}$. Eine vereinzelte Studie zeigte sich radikaler und verlangte das Verbot der Einmanngesellschaften und die Einführung einer zusätzlichen Garantiehaftung für die Gesellschafter ${ }^{38}$. Letztlich setzte der Ausbruch des Ersten Weltkriegs der Debatte ein Ende ${ }^{39}$.

$\mathrm{Zu}$ Beginn der dreißiger Jahre flammten die Reformdiskussionen erneut auf. Sowohl ordoliberale ${ }^{40}$ und als auch dem nationalsozialistischen Gedankengut verschriebene Kritiker verlangten teilweise ausdrücklich die Abschaffung der $\mathrm{GmbH}$ oder ihre völlige Umgestaltung. Begründet wurde dies hauptsächlich mit einer missbräuchlichen Nutzung der GmbH. Sie sei ein typisches Produkt des liberalen Rechtsdenkens des 19. Jahrhunderts und sei nicht mit nationalsozialistischen Anschauungen zu vereinbaren, weil die Haftungsbeschränkung den Unternehmer aus der Haftung entlasse und die "Anonymität” der $\mathrm{GmbH}$ rechtspolitisch unerwünscht sei $^{41}$. Obwohl die Diskussion auf eine Abschaffung der $\mathrm{GmbH}$ hindeutete, wurde dieser Weg im Referentenentwurf von 1939 über eine Reform des GmbH-Gesetzes nicht beschritten. Allerdings lautete es in der Entwurfsbegründung, dass die $\mathrm{GmbH}$ einer „tiefgreifenden Umgestaltung“ bedürfe. Die Leitung der Gesellschaft sollte sich am „allgemeinen Nutzen von Volk und Reich” orientieren, bedeutende Umgestaltungen waren für den Bereich des Gläubigerschutzes vorgesehen ${ }^{42}$. Nicht geklärt ist, warum der Entwurf unter Abkehr der bisherigen ideologischen Linie für die Beibehaltung der $\mathrm{GmbH}$ plädierte. Wahrscheinlich spielte hier eine Rolle, dass der damalige Partei- und Staatsapparat auf die GmbH als wirtschaftlich nützliches Instrument nicht verzichten wollte und konnte ${ }^{43}$. Auch in diesem Fall kam es nicht zur Verwirklichung der Reform, der Zweite Weltkrieg verhinderte entsprechende Maßnahmen ${ }^{44}$.

Weitere umfassende Reformbemühungen waren seit Mitte der sechziger Jahre von der Bundesregierung als Ergänzung der Aktienrechtsnovelle von

36 M. Hachenburg, Bedarf die Gesellschaft mit beschränkter Haftung einer Reform?, Leipziger Zeitschrift für Handels-, Konkurs- und Versicherungsrecht, 1909, 13, 29.

37 Überblick in MünchKommGmbHG/Fleischer, Einl., Rn 84.

38 F. Fränkel, Die GmbH, Eine volkswirtschaftliche Studie, Verlag C.B. Mohr, Tübingen, 1915, S. 250 ff.

39 MünchKommGmbHG/Fleischer, Einl., Rn 86.

40 Vgl. vor allem die Kritik von Hans Grossmann-Doerth, Gutachten für den 5. Deutschen Juristentag in der Tschechoslowakei, 1931, S. 165, 170.

41 F. Klausing, Die Neuordnung der Gesellschaft mit beschränkter Haftung, Erster und Zweiter Arbeitsbericht des GmbH-Ausschusses der Akademie für Deutsches Recht zur Reform der GmbH, Breidenstein, Frankfurt am Main, 1938 und 1940.

42 Vgl. die Regierungsbegründung bei: Werner Schubert (Herausgeber), Entwurf des Reichsjustizministeriums zu einem Gesetz über Gesellschaften mit beschränkter Haftung von 1939, Verlagsgesellschaft Recht und Wirtschaft, Heidelberg, 1985, S. 148 ff.

43 MünchKomm GmbHG/Fleischer, Einl., Rn 93.

44 K. Schmidt, Gesellschaftsrecht, S. 988 f. 
1965 ins Auge gefasst worden. Auch diese "große” Reform des GmbH-Gesetzes sollte zum Scheitern verurteilt sein. Inhaltlich sahen der Referentenentwurf von $1969^{45}$ und der Regierungsentwurf von $1973^{46}$ ein rundum erneuertes GmbH-Gesetz vor, das sehr stark an der Aktiengesellschaft orientiert war. Beabsichtigt war, den Gläubigerschutz zu verstärken, den Minderheitenschutz $\mathrm{zu}$ verbessern, Richterrecht zu kodifizieren und die Rechnungslegung neu zu ordnen. Das Schrifttum sparte indes nicht mit Kritik an diesen Entwürfen ${ }^{47}$. Man warf den Verfassern vor, sich zu sehr vom Aktienrecht und dessen strenger Durchregulierung habe beeinflussen lassen. Die Einschränkung der Gestaltungsfreiheit der gesellschaftsrechtlichen Beziehungen der $\mathrm{GmbH}$ würde diese Rechtsform für kleine und mittlere Unternehmen weniger zugänglich machen. Vermisst wurde auch eine gesellschaftsrechtliche Gesamtkonzeption. Aufgrund des vorzeitigen Endes der damaligen Legislaturperiode des 6. Deutschen Bundestages und wegen anderer gesetzgeberischer Prioritäten wurde der Regierungsentwurf nach nur einer Lesung schließlich fallen gelassen $^{48}$.

Infolge der über weite Strecken des 20. Jahrhunderts nicht realisierten Reformen ist die Weiterentwicklung des Rechts der $\mathrm{GmbH}$ maßgeblich auf die höchstrichterliche Rechtsprechung zurückzuführen ${ }^{49}$. Bezeichnenderweise spricht man bei der Geschichte des GmbH-Rechts von einer Geschichte der Rechtsfortbildung ${ }^{50}$. Die Beurteilung des $\mathrm{GmbH}$-Rechts lag zunächst beim Reichsgericht ${ }^{51}$, später beim Bundesgerichtshof, der sich bemühte, seine Entscheidungen in Kohärenz zu seinem Vorgänger zu gestalten. Ab den achtziger Jahren wurde der Bundesgerichtshof immer häufiger mit dem GmbH-Recht befasst, was zum einen auf eine stetig wachsende Anzahl von Gesellschaften mit beschränkter Haftung, zum anderen auf eine Überalterung des geltenden Gesetzesrechts zurückzuführen war, das der Bundesgerichtshof behutsam den sich wandelnden rechtlichen und wirtschaftlichen Bedingungen anpasste. In Bezug auf die verschiedenen Sachthemen bildeten einen Schwerpunkt seiner Rechtsprechung Fragen der Kapitalaufbringung und -erhaltung ${ }^{52}$.

\section{b) Gesetzesreformen}

Anstatt wie Anfang der siebziger Jahre eine grundlegende Neuregelung des $\mathrm{GmbH}$-Gesetzes durchführen zu wollen, verfolgte die Bundesregierung

45 Bundesministerium der Justiz, Referentenentwurf eines Gesetzes über Gesellschaften mit beschränkter Haftung, 1969.

46 Bundestagsdrucksache zum Regierungsentwurf, 7/253.

47 Übersicht zur Kritik in MünchKommGmbHG/Fleischer, Einl., Rn 104 ff.

48 MünchKommGmbHG/Fleischer, Einl., Rn 107.

49 Daneben spielten auch Vertragspraxis und Rechtswissenschaft eine Rolle.

50 K. Schmidt, Gesellschaftsrecht, S. 987-988.

51 Zur Rechtsprechung des Reichsgerichts, siehe W. Schubert, GmbHG 1892, S. 37 ff.

52 MünchKommGmbHG/Fleischer, Einl., Rn 125 ff. 
mit einem Entwurf aus dem Jahre 1977 die Absicht, lediglich die wesentlichen Missstände im Recht der $\mathrm{GmbH}$ zu beseitigen und nur die notwendigsten Regeln im $\mathrm{GmbH}-\mathrm{Gesetz}$ selbst festzuschreiben ${ }^{53}$. Gemä $\beta$ der Entwurfsbegründung ging es um verschiedene Maßnahmen zur Verbesserung des Gläubigerschutzes wie etwa die Erhöhung des Mindeststammkapitals von 20.000 DM auf 50.000 DM, die Erhöhung der Mindesteinzahlung auf das Stammkapital und verschärfte Prüfungspflichten des Gerichts bei der Anmeldung der GmbH zum Handelsregister, um den Ausbau der Rechte der Minderheitsgesellschafter und um die gesetzliche Verankerung der Ein-Mann-Gründung. Dieser „kleinen GmbH-Reform” war ein anderes Schicksal beschieden als ihren glücklosen Vorgängerinnen, sie wurde im Jahr 1980 verabschiedet $^{54}$. Im Schrifttum ${ }^{55}$ fand die Reform ein geteiltes Echo, in dogmatischer Hinsicht hat sich durch die Neuerungen die GmbH vom historischen Leitbild der Aktiengesellschaft entfernt ${ }^{56}$.

Keine Reform im klassischen Sinn, aber doch eine erhebliche Rechtsanpassung, war zehn Jahre später, am 1. Juli 1990, das Inkraftsetzen des bundesdeutschen $\mathrm{GmbH}$-Rechts in der DDR. In der DDR hatte nach dem Zweiten Weltkrieg das $\mathrm{GmbH}-\mathrm{Gesetz}$ fortbestanden, unmittelbare textliche Veränderungen waren seitdem nicht vorgenommen worden. Die Einführung bundesdeutschen Rechts vollzog sich unter der übergangsweisen Anwendung einiger Sonderregeln ${ }^{57}$.

$\mathrm{Au} \beta \mathrm{er}$ durch die GmbH-Novelle von 1980 wurde das GmbH-Gesetz durch andere gesellschaftsrechtlich relevante Reformgesetze beeinflusst, die jedoch keine Auswirkungen auf die grundlegende Struktur des GmbH-Rechts hatten. $\mathrm{Zu}$ nennen ist hier etwa das Publizitätsgesetz, das Bilanzrichtliniengesetz, das Mitbestimmungsgesetz, das Umwandlungsgesetz, die Insolvenzordnung oder das Gesetz zur Kontrolle und Transparenz im Unternehmensbereich. Teilweise wurden die betreffenden Gesetze im Zuge der Umsetzung von europäischen Richtlinien verabschiedet, wobei deren Einfluss zwar geringer war als im Aktienrecht, aber dennoch in bestimmten Fällen sich spürbar auf das deutsche $\mathrm{GmbH}$-Recht ausgewirkt haben. In diesem Zusammenhang sind zu zitieren

53 Regierungsentwurf über ein Gesetz zur Änderung eines GmbH-Gesetzes und anderer handelsrechtlicher Vorschriften, Bundestagsdrucksache, 7, 1347, S. 27.

54 BGBl. (Bundesgesetzblatt) I, S. 836

55 Zur Kritik im Schrifttum vgl. z.B.: Karl-Friedrich Deutler, Peter Ulmer, Karsten Schmidt, Das neue GmbH-Recht in der Diskussion, Verlag Dr. Otto Schmidt, Köln, 1981; W. Timm, Das neue GmbH-Recht in der Diskussion, GmbH-Rundschau, 1980, S. 286. P. Hommelhoff, Die deutsche GmbH im System der Kapitalgesellschaften, in Roth (Herausgeber): Das System der Kapitalgesellschaften im Umbruch - ein internationaler Vergleich, Verlag Dr. Otto Schmidt, 1990, S. 26,57 f.

56 P. Hommelhoff, Die deutsche GmbH im System der Kapitalgesellschaften, in Roth (Herausgeber): Das System der Kapitalgesellschaften im Umbruch - ein internationaler Vergleich, Verlag Dr. Otto Schmidt, 1990, S. 26, 57 f.

57 Münch. Handbuch, GmbH/Grziwotz, § 1, Rn 26. 
die 1968 verabschiedete und mehrfach geänderte Publikationsrichtlinie e $^{58}$ mit den Regelungen zu Informationspflichten und der Beschränkung von Nichtigkeitsgründen, die Zweigniederlassungsrichtlinie ${ }^{59}$ und die verschiedenen Richtlinien zum Bilanzrecht ${ }^{60}$. Hingegen erforderte die in erster Linie auf die $\mathrm{GmbH}$ anwendbare Einpersonengesellschaftsrichtlinie ${ }^{61}$ aus dem Jahr 1989 für das deutsche Recht keine wesentlichen Anpassungen.

Bedeutsam für die $\mathrm{GmbH}$ war und ist zudem die Rechtsprechung des Europäischen Gerichtshofes zur Niederlassungsfreiheit. Sie hatte erhebliche Auswirkungen auf das deutsche Gesellschaftsrecht im Allgemeinen und veranlasste den Gesetzgeber zu grundlegenden Reformen.

\section{Die jüngste Rechtsentwicklung und die aktuelle Bedeutung der $\mathrm{GmbH}$}

\section{a) Gesetz zur Modernisierung des GmbH-Rechts und zur Bekämpfung von Missbräuchen}

Eine zentrale Reform fand in jüngster Zeit durch das Gesetz zur Modernisierung des $\mathrm{GmbH}$-Rechts und zur Bekämpfung von Missbräuchen - MoMiG vom 23. Oktober 2008 statt $^{62}$. Einige Jahre zuvor war erneut eine Diskussion um die Reformbedürftigkeit der $\mathrm{GmbH}$ aufgekommen ${ }^{63}$. Im Regierungsentwurf vom Mai 2007 über ein Gesetz zur Modernisierung des GmbH-Rechts werden hauptsächlich zwei Gründe für eine erneute Überarbeitung genannt. Einerseits

58 Erste Richtlinie 68/51/EWG des Rates vom 9. März 1968 zur Koordinierung der Schutzbestimmungen, die in den Mitgliedstaaten den Gesellschaften im Sinne des Artikels 58 Absatz 2 des Vertrages im Interesse der Gesellschafter sowie Dritter vorgeschrieben sind, um diese Bestimmungen gleichwertig zu gestalten, ABl. L 65 vom 14.03.1968, S. 8.

59 Elfte Richtlinie 89/666/EWG des Rates vom 21. Dezember 1989 über die Offenlegung von Zweigniederlassungen, die in einem Mitgliedstaat von Gesellschaften bestimmter Rechtsformen errichtet wurden, die dem Recht eines anderen Staates unterliegen, Abl. L 395 vom 30.12.1989, S. 36.

60 Vierte Richtlinie 78/660/EWG des Rates vom 25. Juli 1978 aufgrund von Artikel 54 Absatz 3 Buchstabe $\mathrm{g}$ ) des Vertrages über den Jahresabschluss von Gesellschaften bestimmter Rechtsformen, ABl. L 222 vom 25.4.1978, S. 11; Siebente Richtlinie 83/349/EWG des Rates vom 13. Juni 1983 aufgrund von Artikel 54 Absatz 3 Buchstabe g des Vertrags über den konsolidierten Abschluss, ABl. L 193 vom 18.07.1983, S. 1; Richtlinie 2006/43/EG des Europäischen Parlaments und des Rates vom 17. Mai 2006 über Abschlussprüfungen von Jahresabschlüssen und konsolidierten Abschlüssen, zur Änderung der Richtlinien 78/660/EWG und 83/349/EWG des Rates und zur Aufhebung der Richtlinie 84/253/EWG des Rates, ABl. L 157, 9.06.2006, S. 87.

61 Zwölfte Richtlinie 89/667/EWG des Rates vom 21. Dezember 1989 auf dem Gebiet des Gesellschaftsrechts betreffend Gesellschaften mit beschränkter Haftung mit einem einzigen Gesellschafter ABl. L 395, vom 30.12.1989, S. 40.

62 BGBl. (Bundesgesetzblatt) I, S. 2026.

63 Siehe zum Gang der Diskussion: MünchKommGmbHG/FLeISCHER, Einl., Rn 116, 117. 
sollte die $\mathrm{GmbH}$, wie schon in der Vergangenheit, besser gegen Missbräuche gerade auch im Hinblick auf die zunehmenden Insolvenzen geschützt werden ${ }^{64}$.

Andererseits ging das Streben des Gesetzgebers dahin, das GmbH-Recht zu modernisieren und zu deregulieren. Anlass dafür war vor allem der zunehmende Wettbewerb im europäischen Gesellschaftsrecht. Namentlich die Rechtsprechung des Europäischen Gerichtshofes zur Niederlassungsfreiheit von Kapitalgesellschaften hatte in Deutschland zu Zweifeln an der Konkurrenzfähigkeit der $\mathrm{GmbH}$ geführt. Aufgrund der Entscheidungen in den Rechtssachen Centros $^{65}$, Überseering ${ }^{66}$ und Inspire Art ${ }^{67}$ wurde es möglich, Auslandsgesellschaften mit tatsächlichem Sitz im Inland ohne gesetzliches Mindeststammkapital und die damit einhergehenden nach GmbH-Recht vorgesehenen komplizierten Kapitalaufbringungsregeln $\mathrm{zu}$ gründen $^{68}$. Eine solche Vorgehensweise war bis dato in Deutschland aufgrund der traditionell geltenden Sitztheorie als international privatrechtlicher Anknüpfungspunkt für das auf die Gesellschaft anwendbare Recht nicht möglich gewesen. Gemä $\beta$ der Sitztheorie richtet sich das Gesellschaftsstatut nach dem Recht des Staates, wo die Gesellschaft ihren Hauptverwaltungssitz hat ${ }^{69}$. Eine in Goßbritannien gegründete „limited” hätte danach bei Verlegung des tatsächlichen Sitzes nach Deutschland, den Vorgaben des GmbH-Gesetzes entsprechen müssen. Der Europäische Gerichtshof hatte nun aber diese Auffassung als unvereinbar mit der Niederlassungsfreiheit erklärt.

Dies bewirkte im Weiteren, dass die englische „limited”, die seit Entstehung des GmbH-Gesetzes Ende des 19. Jahrhundert in Deutschland in Vergessenheit geraten war, aufgrund ihrer immer noch währenden einfachen Gründungsvorschriften wieder in den Blickpunkt der deutschen Rechtspolitik rückte. Wie seit Bekanntgabe der Urteile zu erwarten gewesen war, avancierte die „limited” binnen weniger Jahre zu einer äußerst beliebten Rechtsform für deutsche Unternehmensgründer und steht seitdem gleichberechtigt als Rechtsform der deutschen $\mathrm{GmbH}$ im Inland gegenüber.

Als Reaktion auf diese Entwicklung wurde durch das Gesetz zur Modernisierung des GmbH-Rechts und zur Bekämpfung von Missbräuchen eine Reihe von zahlreichen Neuregelungen beschlossen, die die Wettbewerbsfähigkeit

64 Begründung zum Entwurf eines Gesetzes zur Modernisierung des GmbH-Rechts und zur Bekämpfung von Missbräuchen (MoMiG), Bundestagsdrucksache, 16/6140, S. 58.

65 EuGH Urteil vom 9.3.1999 - Rechtssache C-212/97, Sammlung 1999 I -1459.

66 EuGH Urteil vom 5.11.2002 - Rechtssache C-208/00, Sammlung 2002 I - 9919.

67 EuGH Urteil vom 30.9.2003 - Rechtssache C-167/01, Sammlung 2003 I - 10155.

68 Baumbach/Hueck, GmbHG, Hueck/Fastrich, Einl., Rn 38.

69 Zurzeit gilt in Deutschland noch die Sitztheorie. Es wird aber erwogen, zukünftig im Rahmen des Internationalen Privatrechts an die Gründungstheorie anzuknüpfen. 
der $\mathrm{GmbH}$ verbessern sollten, wobei hier der Gesetzgeber den Zielkonflikt zwischen vereinfachenden Regeln und Missbrauchsverhinderung bewältigen musste $^{70}$. Deutlich wird dies bei den Regelungen über die Kapitalaufbringung und Kapitalerhaltung. Der Gesetzgeber, nach ersten Überlegungen zur Herabsetzung des Stammkapitals auf 1 Euro, hat es letztlich für den Fall der klassischen $\mathrm{GmbH}$ bei einem Mindeststammkapital von 25000 Euro belassen. Anderes gilt aber nunmehr für den Fall der neu eingeführten Unternehmergesellschaft, einer speziellen Form der $\mathrm{GmbH}$, die fast ganz ohne Mindeststammkapital gegründet werden kann ${ }^{71}$. Im Gegenzug darf der erwirtschaftete Gewinn nicht voll ausgeschüttet werden, solange bis die Gesellschaft das Mindeststammkapital von 25000 Euro angespart hat. Nichtsdestoweniger ist hier aber das der GmbH zugrunde liegende Gesamtkonzept, wonach die zur Zeit der Eintragung notwendige Mindestkapitalausstattung die notwendige Entsprechung für die Beschränkung der Haftung auf das Gesellschaftsvermögen darstellt, in Frage gestellt worden. Damit entfernt sich der Gläubigerschutz von der präventiven Kapitalaufbringungshaftung und ersetzt diese durch spätere Verhaltensstandards von Gesellschaftern und Geschäftsführern, vor allem vor und während einer Insolvenz $z^{72}$.

Eine direkte Antwort und ein Novum des deutschen GmbH-Gesetzes auf die europäische Rechtsprechung zur Niederlassungsfreiheit ist zudem, dass eine in Deutschland gegründete $\mathrm{GmbH}$ ihren tatsächlichen Sitz ins Ausland verlegen kann, ohne dass sie ihre nationale Identität verliert. Diese Neuregelung und die Einführung der Unternehmergesellschaft sind nur ein kleiner Auszug aus dem umfangreichen Gesetzespaket, das als bedeutsamste Reform seit dem Inkrafttreten des $\mathrm{GmbH}-\mathrm{Gesetzes}$ bezeichnet werden kann. Die Kritik hat das Gesetz eher positiv beurteilt, auch im Hinblick darauf, dass der Gesetzgeber die Grundstruktur des Gesetzes intakt gelassen und einer schrittweisen Verjüngung den Vorzug gegeben hat ${ }^{73}$.

\section{b) Erscheinungsformen und Verbreitung der GmbH}

Der historische Gesetzgeber hatte eine einheitliche Form der $\mathrm{GmbH}$ vorgesehen. Danach ist die $\mathrm{GmbH}$ eine mit eigener Rechtspersönlichkeit ausgestattete Gesellschaft, bei der die Haftung gegenüber ihren Gläubigern auf das Gesellschaftskapital beschränkt ist und die ein in Stammeinlagen zerlegtes

70 Vgl. dazu Karsten Schmidt, GmbH-Reform auf Kosten der Geschäftsführer? Zum (Un-)Gleichgewicht Gesellschafterrisiko und Geschäftsführerrisiko im Entwurf eines MoMiG und in der BGH-Recht-sprechung, GmbHR 2008, S. 449.

71 Vgl. den neuen $₫ 5 \mathrm{a}$ GmbHG. Die Gründung kann bereits mit einem Stammkapital von 1 Euro erfolgen.

72 Dazu Baumbach/Hueck, GmbHG, Hueck/Fastrich, Einl., Rn 52, 53.

73 So MünchKomm GmbHG/Fleischer, Einl., Rn 120 f. mit weiteren Nachweisen; vgl. auch Baumbach/ Hueck, GmbHG, Hueck/Fastrich, Einl., Rn 47. 
Stammkapital aufweist. Im Laufe der Zeit haben sich ausgehend von diesem Modell verschiedene Erscheinungsformen der $\mathrm{GmbH}$ etabliert.

Je nachdem, wie sich der Gesellschafterkreis der Gesellschaft zusammensetzt, spricht man von einer personalistisch oder kapitalistisch geprägten $\mathrm{GmbH}$. Erstere zeichnet sich durch einen kleinen Kreis von Gesellschaftern aus, die sich kennen und als Geschäftsführer oder Angestellte im Unternehmen mitarbeiten. So haben über die Hälfte aller Gesellschaften mit beschränkter Haftung nur zwei bis fünf Gesellschafter. Durch die Vinkulierung der Anteile und Vorkaufsrechte, wird die Anzahl der Gesellschafter beschränkt. Typischerweise findet man hier kleine oder mittlere Unternehmen, wobei sich häufig mittelständische Familienbetriebe dieser Gesellschaftsform bedienen. Hingegen steht bei der GmbH kapitalistischen Zuschnitts die Vermögensbeteiligung im Vordergrund. Die Gesellschafter sind häufig juristische Personen und in der Regel handelt es sich um Großunternehmen. Die GmbH wird hier jedoch relativ selten verwendet, gewöhnlich wird die Aktiengesellschaft vorgezogen. Eine Ausnahme besteht bei der Konzernbildung, für die die $\mathrm{GmbH}$ als prädestiniert gilt. Einen Sonderfall bilden die Einpersonen-Gesellschaften, die bereits 1904 bei der nachträglichen Vereinigung der Anteile in einer Hand von der Rechtsprechung anerkannt wurden und deren Gründung seit der Novelle von 1980 zugelassen ist.

Die GmbH kommt nicht nur in ihrer ursprünglichen Form vor, sondern wird gerne mit anderen Rechtsformen verbunden. Eine beliebte Form dieser Grundtypenvermischung ist die $\mathrm{GmbH}$ \& Co.KG, bei der im Unterschied zur üblichen Kommanditgesellschaft keine natürliche Person sondern die $\mathrm{GmbH}$ als Komplementärin auftritt.

Schließlich lassen sich die Gesellschaften mit beschränkter Haftung auch nach dem von ihnen verfolgten Zweck unterscheiden. Die GmbH kann, was auch historisch so angelegt war, grundsätzlich für jeden Zweck eingesetzt werden, ist also nicht auf eine wirtschaftliche Verwendung festgelegt, sondern kann auch ideelle Zwecke erfüllen, wobei Gesellschaften mit erwerbswirtschaftlicher Zielsetzung die große Mehrzahl bilden.

Die Zahl der in Deutschland gegründeten Gesellschaften mit beschränkter Haftung hat seit ihrer Einführung ständig zugenommen. Verzeichnete man 1950 noch 22.000 Gesellschaften so ergibt sich nach Schätzungen ein heutiger Stand von etwa 1 Million Gesellschaften mit beschränkter Haftung. Im Vergleich dazu gab es zum 1. Januar 2008 nur etwas über 18.000 Aktiengesellschaften und um die 15.000 ,private limited companies”. Insgesamt stellt die GmbH in Deutschland die mit Abstand am weitesten verbreitete Gesellschaftsform dar ${ }^{74}$.

74 Vgl. zum Zahlenmaterial Baumbach/Hueck, GmbHG, Hueck/Fastrich, Einl., Rn 15 ff. und die sehr umfangreichen Angaben in: MünchKommGmbHG/Fleischer, Einl., Rn 198 ff., 219. Teilweise ist wegen fehlender oder schwierig zu erstellender Statistiken von Schätzungen auszugehen. 


\section{Schlussbetrachtung}

Die GmbH kann heute auf eine über 100-jährige Erfolgsgeschichte zurückblicken. Sie ist vom historischen Gesetzgeber zur Erfüllung bestimmter rechts- und wirtschaftspolitischer Zwecke geschaffen worden und konnte die in sie gesetzten Erwartungen voll erfüllen oder sogar übertreffen. Trotz ihrer Schwächen zeichnet sich ihre Entwicklung weniger durch systembetreffende Veränderungen als vielmehr durch eine schrittweise Anpassung an die Erfordernisse des modernen Wirtschaftslebens aus. Die Gründe für ihre Einführung wie auch für ihre jüngste Neuregelung basieren in weiten Teilen auf gleichartigen Überlegungen, nämlich der Schaffung bzw. der Beibehaltung einer effizienten und wettbewerbsfähigen Gesellschaftsform als Reaktion auf die rechtlichen und tatsächlichen Gegebenheiten der jeweiligen Epoche. Zusammenfassend lässt sich feststellen, dass die GmbH heute eine lebendige und flexible Rechtsform darstellt, die sich für die Wirtschaftspraxis als unerlässlich erwiesen hat.

\section{Bibliography}

Ballerstedt, Kurt, 75 Jahre GmbH-Gesetz, GmbHR (GmbH-Rundschau), 1978, 66.

Baumbach, Adolf, Hueck, Alfred GmbHG, Gesetz betreffend die Gesellschaften mit beschränkter Haftung, 19. Auflage, Verlag C.H. Beck, München, 2010.

Deutler, Karl-Friedrich, Ulmer, Peter, Schmidt, Karsten, Das neue GmbH-Recht in der Diskussion, Verlag Dr. Otto Schmidt, Köln, 1981.

Fränkel, Franz, Die GmbH, Eine volkswirtschaftliche Studie, Verlag C.B. Mohr, Tübingen, 1915.

Grossmann-Doerth, Hans, Reform des Gesetzes betreffend die Gesellschaften mit beschränkter Haftung, Gutachten für den 5. Deutschen Juristentag in der Tschechoslowakei, 1931.

Hachenburg, Max, Bedarf die Gesellschaft mit beschränkter Haftung einer Reform?, Leipziger Zeitschrift für Handels-, Konkurs- und Versicherungsrecht, 1909, 13.

Hommelhoff, Peter, Die deutsche GmbH im System der Kapitalgesellschaften, in Roth (Herausgeber): Das System der Kapitalgesellschaften im Umbruch - ein internationaler Vergleich, Verlag Dr. Otto Schmidt, 1990, 26. 
Klausing, Friedrich, Die Neuordnung der Gesellschaft mit beschränkter Haftung, Erster und Zweiter Arbeitsbericht des GmbH-Ausschusses der Akademie für Deutsches Recht zur Reform der GmbH, Breidenstein, Frankfurt am Main, 1938 und 1940.

Meyer, Justus Haftungsbeschränkung im Recht der Handelsgesellschaften, Springer-Verlag Berlin, Heidelberg, New York, 2000.

Münchener Handbuch des Gesellschaftsrechts, Band 3, Gesellschaft mit beschränkter Haftung, herausgegeben von Hans-Joachim Priester und Dieter Mayer, Verlag C.H. Beck München 2003.

Münchener Kommentar zum Gesetz Betreffend die Gesellschaft mit beschränkter Haftung - GmbHG, Band 1, \$\$ 1-34, herausgegeben von Holger Fleischer und Wulf Goette, Verlag C.H. Beck, München 2010.

Schilling, Wolfgang, Rechtspolitische Gedanken zur GmbH \& Co., [in:]Festgabe für Otto Kunze, Recht und Rechtsleben in der sozialen Demokratie, Duncker \& Humblot, Berlin, 1969, 189.

Schmidt, Karsten, Gesellschaftsrecht, 4. Auflage, Carl Heymanns Verlag, Köln, Bonn, Berlin, München 2002.

Schmidt, Karsten, GmbH-Reform auf Kosten der Geschäftsführer? Zum (Un-) Gleichgewicht Gesellschafterrisiko und Geschäftsführerrisiko im Entwurf eines MoMiG und in der BGH-Rechtsprechung, GmbHR 2008, S. 449.

Schubert, Werner Das GmbH-Gesetz von 1892 „eine Zierde unserer Reichsgesetzsammlung", in: Festschrift 100 Jahre GmbH-Gesetz, herausgegeben von Marcus Lutter, Peter Ulmer, Wolfgang Zöllner, Verlag Dr. Otto Schmidt, Köln, 1992, 4.

Timm, Wolfgang, Das neue GmbH-Recht in der Diskussion, GmbH-Rundschau. 1980, 286. 